\title{
Trapped acoustic waves in the potential core of subsonic jets
}

\author{
Aaron Towne*广 André V. G. Cavalierił Peter Jordan\$ Tim Colonius, \\ Vincent Jaunet, Oliver T. Schmidt,* Guillaume A. Brès ${ }^{\dagger \dagger}$
}

\begin{abstract}
The purpose of this paper is to characterize and model waves that are observed within the potential core of subsonic jets and that have been previously detected as tones in the near-nozzle region. Using three models (the linearized Euler equations, a cylindrical vortex sheet, and a cylindrical duct with pressure release boundary conditions), we show that these waves can be described by linear modes of the jet and correspond to acoustic waves that are trapped within the potential core. At certain frequencies, these trapped waves resonate due to repeated reflection between end conditions provided by the nozzle and the streamwise contraction of the potential core. Our models accurately capture numerous aspects the potential core waves that are extracted from large-eddy-simulation data of a Mach 0.9 isothermal jet. Furthermore, the vortex sheet model indicates that this behavior is possible for only a limited range of Mach numbers that is consistent with previous experimental observations.
\end{abstract}

\section{Introduction}

Simulations of jets with high subsonic Mach numbers show the presence of negative phase-velocity waves in the jet potential core. For example, figure 1 shows an instantaneous snapshot of the axisymmetric component of the pressure field from a recent large-eddy-simulation of a Mach 0.9 isothermal, turbulent jet by Brès et. al. ${ }^{1,2}$ We will use data from this simulation throughout this paper. The red dashed line shows the location where the mean streamwise velocity equals $99 \%$ of the jet velocity, which acts as a proxy for the outer edge of the potential core. The presence of waves within the first few diameters of the potential core is clear. The upstream phase velocity of these waves can be confirmed by examining the frequency-wavenumber decomposition of the data in the potential core. For example, the decomposition of the pressure along the polar axis for the axisymmetric component $(m=0)$ and along $r / D=0.1$ for the helical component $(m=1)$ is shown in figure 2 . The bands of energy at negative wavenumbers match the frequencies and wavelengths observed for the waves in the potential core.

These waves produce discrete spectral peaks, or tones, that can be observed outside of the potential core in the near-nozzle region. For example, the power-spectral-density of the first two azimuthal modes of the pressure signals at $(x / D, r / D)=(0.02,0.8)$ are plotted in figure 3 . Data from a complimentary experiment ${ }^{2}$ is shown in addition to the LES data. Similar tones were observed by Suzuki \& Colonius ${ }^{3}$ for certain subsonic operating conditions (but not others) and attributed to an internal aerodynamic resonance in the upstream plumbing. Sinha ${ }^{4}$ also observed similar tones and attributed them to debris that was later detected within the internal jet plumbing, but this hypothesis was not confirmed. Both of these explanations attribute the tones to extrinsic factors.

The purpose of this paper is to examine and model the waves observed within the potential core and the associated tones. We will show that they are an intrinsic feature of the jet dynamics and can be described

\footnotetext{
*At time of work: Ph.D. student, California Institute of Technology, Pasadena, CA, USA

${ }^{\dagger}$ Currently: Postdoctoral fellow, Center for Turbulence Research, Stanford University, Stanford, CA, USA

¥Assistant Professor, Instituto Tecnolõgico de Aeronáutica, São José dos Campos, Brazil

§Research Scientist, Institut Pprime, CNRS - University of Poitiers - ENSMA, Poitiers, France

IProfessor, California Institute of Technology, Pasadena, CA, USA

$\|$ Postdoc, Institut Pprime, CNRS - University of Poitiers - ENSMA, Poitiers, France

**Postdoc, California Institute of Technology, Pasadena, CA, USA

${ }^{\dagger}$ Senior Research Scientist, CASCADE Technologies, Palo Alto, CA, USA
} 


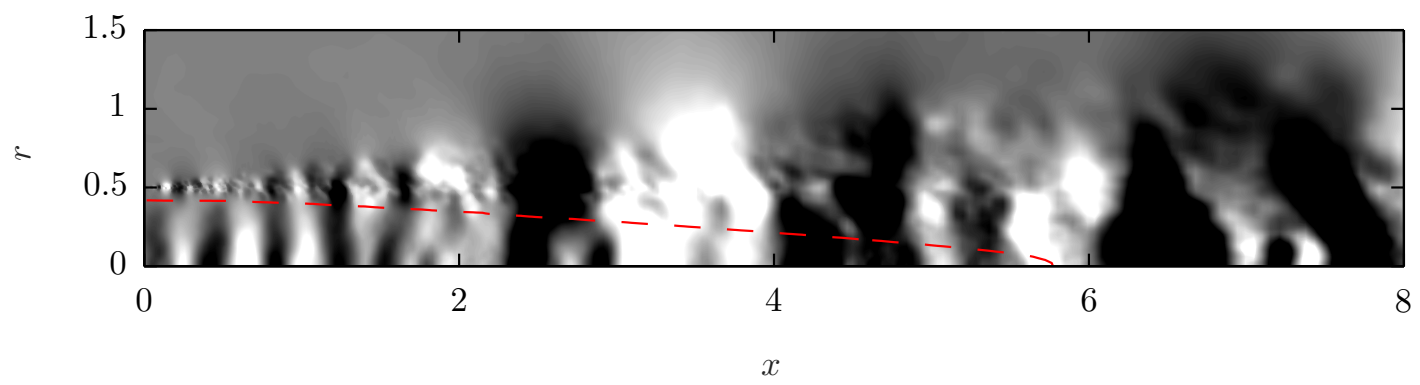

Figure 1: Instantaneous snapshot of the axisymmetric component of the pressure field of a Mach 0.9 jet. The red dashed line shows the location where the mean streamwise velocity equals $99 \%$ of the jet velocity, which acts as a proxy for the outer edge of the potential core.
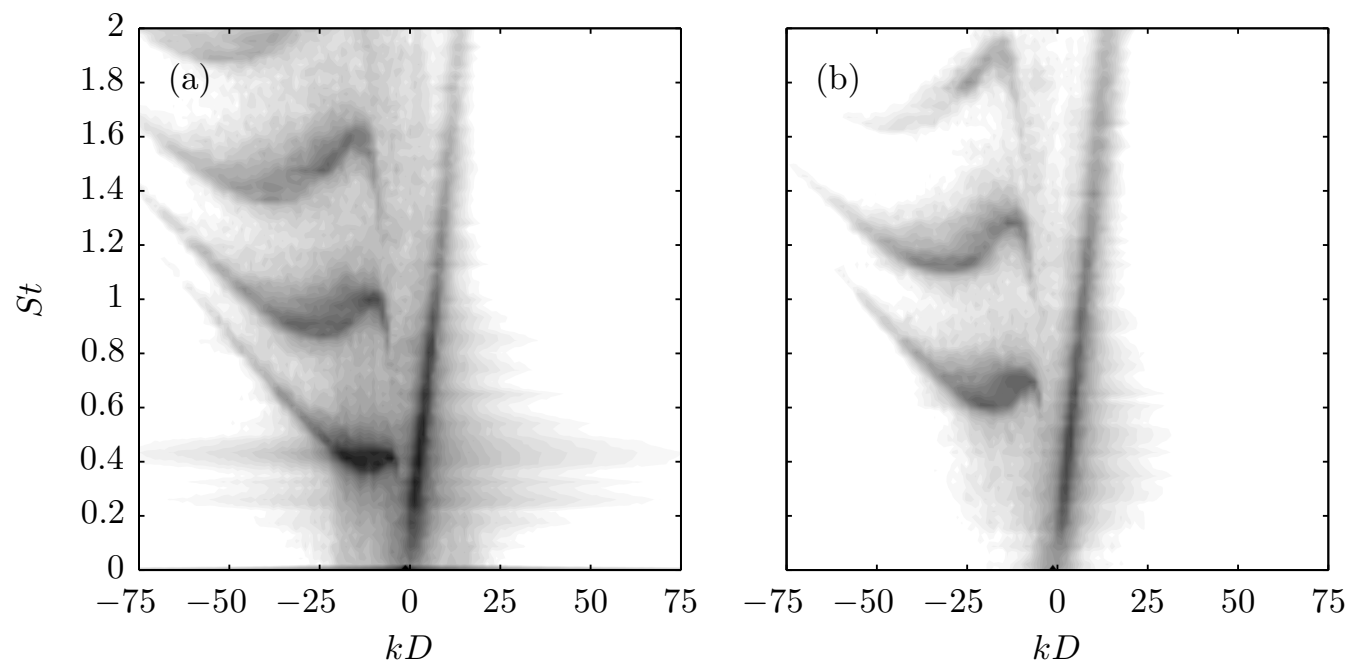

Figure 2: Frequency-wavenumber energy distribution of the pressure in the potential core: (a) $m=0$, $r / D=0$; (b) $m=1, r / D=0.1$. The waves of interest produce the negative-wavenumber bands of energy.

by certain linear instability modes of the jet. Specifically, the relevant modes are members of the "irregular" mode II family identified by Michalke. ${ }^{5}$ These modes were originally deemed unphysical and disregarded by various authors. ${ }^{5-7}$ This position was neatly summarized by Michalke: ${ }^{5}$

The physical meaning of this "irregular perturbation mode", however, is not clarified. Since these modes have not yet been observed in experiments, it is natural to believe that these solutions are a consequence of an infinite parallel flow... Physically speaking, this "irregular" mode II is probably meaningless, as already mentioned.

We will show that certain properties of these modes can be explained by the modes of a cylindrical duct with pressure release boundary conditions. This connection provides insight into the physical mechanisms at play - specifically that the waves under investigation are acoustic waves that are trapped within the potential core. Furthermore, using weakly-nonparallel stability theory, we will show that at certain frequencies, these waves resonant due to repeated reflection between end conditions provided by the nozzle and the streamwise contraction of the potential core.

The remainder of the paper is arranged as follows. In Section II, we introduce the generalized stability theory developed by Briggs ${ }^{8}$ and Bers, ${ }^{9}$ which we use to analyze our models. In Section III, we introduce three models: the linearized Euler equations, a cylindrical vortex sheet and a cylindrical soft duct. Section IV presents parallel-flow results from the models and comparisons with the LES data. In Section V, we inves- 

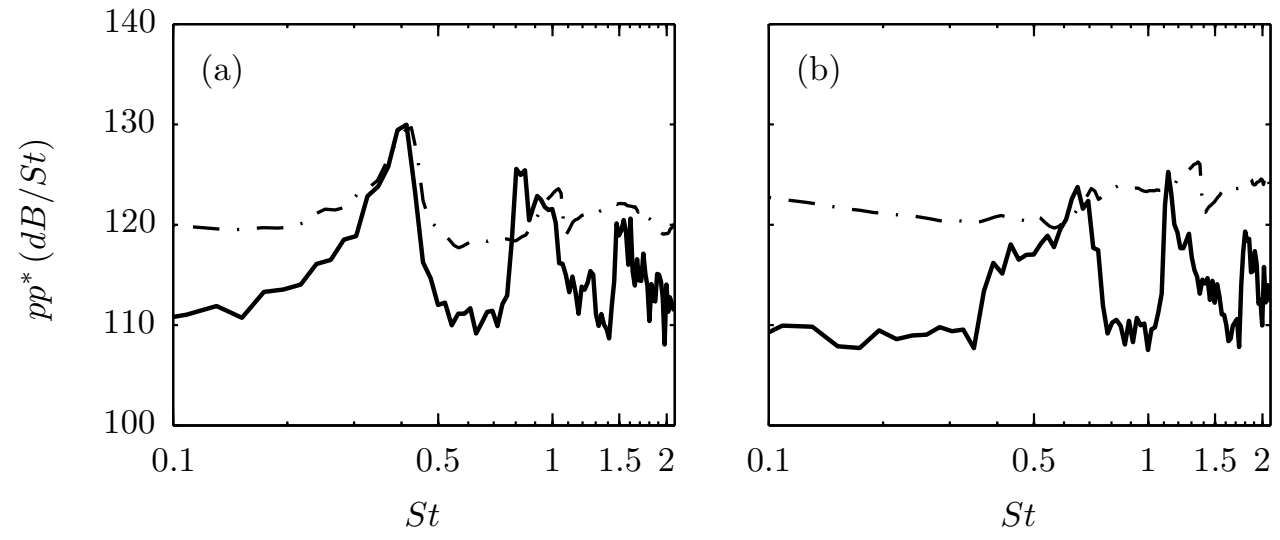

Figure 3: Power-spectral-density of the of pressure in the near-nozzle region $((x / D, r / D)=(0.02,0.8)):(a)$ $m=0$; (b) $m=1$. Lines:(— Brès et. $a l^{1}$ LES data; (- - - - - ) experimental measurements. ${ }^{2}$

tigate the resonance of the trapped waves using the linearized Euler model along with weakly-nonparallel theory. Section VI summarized and concludes the paper.

\section{Briggs-Bers stability theory}

We wish to relate the waves observed in the potential core to the modal properties of the jet. To do so, we will analyze each of the models introduced in the next section using the spatiotemporal stability theory developed by Briggs ${ }^{8}$ and Bers ${ }^{9}$ which is a generalization of the classical concepts of temporal and spatial stability. The theory was originally developed for spatially homogeneous equations (which in the context of fluid dynamics corresponds to strictly parallel flows), but was later generalized to partially account for slow streamwise variation. We introduce the parallel flow theory here and highlight the most salient points of the weakly-nonparallel theory in Section V.

Consider the linear one-dimensional partial differential equation

$$
\mathcal{D}\left(-i \frac{\partial}{\partial x}, i \frac{\partial}{\partial t}\right) q(x, t)=s(x, t) .
$$

The operator does not depend on $x$ or $t$. The following ideas can be extended to multidimensional equations, but we present the one-dimensional case here for simplicity. The solution of equation (1) for any source $s(x, t)$ can be obtained by convolving the source with a Green's function $G(x, t)$ satisfying

$$
\mathcal{D}\left(-i \frac{\partial}{\partial x}, i \frac{\partial}{\partial t}\right) G(x, t)=\delta(x) \delta(t) .
$$

Therefore, the stability characteristics of equation (1) under arbitrary forcing can be determined by analyzing the properties of $G(x, t)$.

The Green's function can be obtained using Fourier and Laplace transforms. Specifically, applying a Fourier transform in $x$ and a Laplace transform in $t$ to equation (2) gives

$$
\mathcal{D}(k, \omega) \hat{G}(k, \omega)=1,
$$

where $k$ and $\omega$ are the Fourier and Laplace duals of $x$ and $t$, respectively, and $\hat{G}(k, \omega)$ is the Fourier-Laplace transform of $G(x, t)$. We have ignored initial transients since the jet is statistically stationary. For future use, we define the expression $\omega=\omega(k)$ to represent a $(k, \omega)$ pair that satisfies the dispersion relation $\mathcal{D}(k, \omega)=0$. Equivalently, the inverse of the expression can be written $k=k(\omega)$.

The Green's function $G(x, t)$ is obtained by solving equation (3) for $\hat{G}(k, \omega)$ and taking the inverse 
transforms:

$$
G(x, t)=\frac{1}{4 \pi^{2}} \int_{L} \int_{F} \frac{1}{\mathcal{D}(k, \omega)} e^{i(k x-\omega t)} d k d \omega .
$$

The stability characteristics of equation (1) can now be evaluated by determining the long-time behavior of the Green's function $G(x, t)$. Of particular interest is the so-called absolute growth rate observed at the source location $x=0$. If the absolute growth rate is negative, then all disturbances move away from their source such that the solution decays at the source location as $t$ becomes large. On the other hand, if the absolute growth rate is positive, disturbances grow at their source location and eventually engulf the entire domain. If the absolute growth rate is zero, disturbances neither grow nor decay but instead persist indefinitely. These three scenarios are termed absolute stability, absolute instability, and neutral (or marginal) stability, respectively.

The absolute growth rate can be obtained by asymptotically evaluating the integrals in equation (4). Beginning with a Laplace contour $L$ with sufficient high $\omega_{i}$ (the subscripts $r$ and $i$ refer to the real and imaginary parts of a variable from here on out), we subsequently lower the Laplace contour by decreasing $\omega_{i}$ until the Fourier contour is pinched between two branches of $k(\omega)$ that correspond to upstream and downstream traveling solutions. The distinction between upstream and downstream modes is based on causality constraints and is determined by the asymptotic behavior of $k(\omega)$ at large $\omega_{i}$ : a mode is downstream traveling if

$$
\lim _{\omega_{i} \rightarrow+\infty} k_{i}=+\infty
$$

and upstream traveling if

$$
\lim _{\omega_{i} \rightarrow+\infty} k_{i}=-\infty .
$$

The Laplace contour cannot be lowered beyond this pinch point, which is denoted $\omega^{0}=\omega\left(k^{0}\right)$, without violating causality. These pinch points are in fact saddle points in the complex $k$-plane and satisfy

$$
\frac{\partial \omega}{\partial k}\left(k^{0}\right)=0
$$

which shows that they represent waves that do not propagate away from their source. Since $\omega^{0}=\omega\left(k^{0}\right)$ represents the pole in the Laplace plane with the highest imaginary part, it dominates the long-time solution and the absolute growth rate is thus given by $\omega_{i}^{0}$.

\section{Models}

We will use three models to explain the waves observed in LES data and explore their Mach number and temperature ratio dependence: the linearized Euler equations, a cylindrical vortex sheet, and a cylindrical soft duct.

\section{III.A. Linearized Euler equations}

The linearized Euler equations can be written

$$
\frac{\partial \boldsymbol{q}^{\prime}}{\partial t}+A_{x} \frac{\partial \boldsymbol{q}^{\prime}}{\partial x}+A_{r} \frac{\partial \boldsymbol{q}^{\prime}}{\partial r}+A_{\theta} \frac{\partial \boldsymbol{q}^{\prime}}{\partial \theta}+A_{0} \boldsymbol{q}^{\prime}=\mathbf{0}
$$

where $\boldsymbol{q}(x, r, \theta, t)=\overline{\boldsymbol{q}}(x, r)+\boldsymbol{q}^{\prime}(x, r, \theta, t)$ is a vector of flow variables and $\boldsymbol{q}^{\prime}$ is a perturbation to the mean flow $\overline{\boldsymbol{q}}$. The matrix coefficients are functions of the mean flow and can be found for example in Towne \& Colonius. ${ }^{10}$

To apply the parallel-flow stability theory, we freeze the mean flow at a particular $x$ location $(x=0$ in Section IV and other values in Section V) and neglect axial derivatives of the mean flow and the mean radial velocity for consistency with the local approximation. Taking Fourier-Laplace transforms of the frozen equations, or equivalently applying the normal modes ansatz

$$
\boldsymbol{q}^{\prime}(x, r, \theta, t)=\hat{\boldsymbol{q}}(r) e^{(i k x+i m \theta-i \omega t)},
$$

yields

$$
\left[-i \omega I+i k A_{x}+A_{r} \frac{\partial}{\partial r}+i m A_{\theta}+A_{0}\right] \hat{\boldsymbol{q}}(r)=0
$$


Since we wish to identify saddle points in the complex $k$-plane, we will specify (real or complex) values for $\omega$ and solve the resulting eigenvalue problem for $k=k(\omega)$ for fixed values of the integer azimuthal wavenumber $m$. The eigenvalue problem is solved numerically by discretizing equation (10) in the radial direction, leading to a matrix eigenvalue problem for $k$. We use fourth-order central finite differences with summation-by-parts boundary closures. ${ }^{11}$ Radiation boundary conditions are enforced using a super-grid damping layer ${ }^{12}$ truncated by characteristic conditions, ${ }^{13}$ and pole conditions are implemented using the scheme of Mohseni \& Colonius. ${ }^{14}$ The eigenvalues and eigenvectors of interest are well-resolved using 200 grid points.

\section{III.B. Cylindrical vortex sheet}

The dispersion relation for a compressible, cylindrical vortex sheet was first derived by Lessen et. al ${ }^{15}$ and has been studied by a number of authors. ${ }^{6,16}$ It can be written schematically as

$$
\mathcal{D}_{j}(k, \omega ; m, M, T)=0
$$

with

$$
\mathcal{D}_{j}=\frac{1}{\left(1-\frac{k M}{\omega}\right)^{2}}+\frac{1}{T} \frac{I_{m}\left(\frac{\lambda_{i}}{2}\right)\left[\frac{\lambda_{o}}{2} K_{m-1}\left(\frac{\lambda_{o}}{2}\right)+m K_{m}\left(\frac{\lambda_{o}}{2}\right)\right]}{K_{m}\left(\frac{\lambda_{o}}{2}\right)\left[\frac{\lambda_{i}}{2} I_{m-1}\left(\frac{\lambda_{i}}{2}\right)-m I_{m}\left(\frac{\lambda_{i}}{2}\right)\right]}
$$

and

$$
\begin{gathered}
\lambda_{i}=\sqrt{k^{2}-\frac{1}{T}(\omega-M k)^{2}}, \\
\lambda_{o}=\sqrt{k^{2}-\omega^{2}} .
\end{gathered}
$$

$I_{m}$ and $K_{m}$ are modified Bessel functions of the first and second kind, respectively. All quantities have been normalized by the jet diameter and far-field thermodynamic quantities such that $M=U_{j e t} / c_{\infty}$ is the acoustic Mach number and $T=T_{j e t} / T_{\infty}$ is the temperature ratio. Equation 11 is satisfied for only certain combinations of the temporal frequency $\omega$ and axial wavenumber $k$, both of which can in general be complex. These special $(k, \omega)$ pairs are the eigenvalues of the vortex sheet.

\section{III.C. Cylindrical soft duct}

The modes of a cylindrical soft duct (a duct with pressure-release boundary conditions at the wall) provide insight into the Euler and vortex sheet modes that are relevant to this investigation. The dispersion relation for the duct can be obtained analytically as

$$
k_{m, n}^{ \pm}=\frac{-\omega M \pm \sqrt{T} \sqrt{\omega^{2}-4\left(T-M^{2}\right) \beta_{m, n}^{2}}}{T-M^{2}},
$$

where $\beta_{m, n}$ is the $n^{\text {th }}$ root of the Bessel function $J_{m}$. The $k_{m, n}^{+}$and $k_{m, n}^{-}$roots can be identified as downstream and upstream traveling, respectively, using equations (5) and (6). These two modes are labelled evanescent if the quantity inside the square root in equation (14) is negative, since they decay exponentially in the direction of propagation; and propagative if the quantity inside the square root is positive, with phase speeds dictated by the wave equation. The switch from evanescent to propagative behaviour, referred-to as the cut-on condition, occurs when the two roots form a saddle point at

$$
\begin{gathered}
\omega_{m, n}^{0}=2 \sqrt{T-M^{2}} \beta_{m, n}, \\
k_{m, n}^{0}=\frac{-2 M}{\sqrt{T-M^{2}}} \beta_{m, n} .
\end{gathered}
$$

The absolute frequency at each saddle has zero imaginary part, so the duct modes are neutrally stable, as one could anticipate from acoustic theory.

The duct modes approximately satisfy the vortex sheet dispersion relation in certain situations. Substituting a $(k, \omega)$ combination that satisfies equation (14) into equation (12) gives

$$
\mathcal{D}_{j}\left(k_{m, n}^{ \pm}, \omega ; m, M, T\right)=\frac{1}{\left(1-\frac{M k_{m, n}^{ \pm}}{\omega}\right)^{2}} .
$$




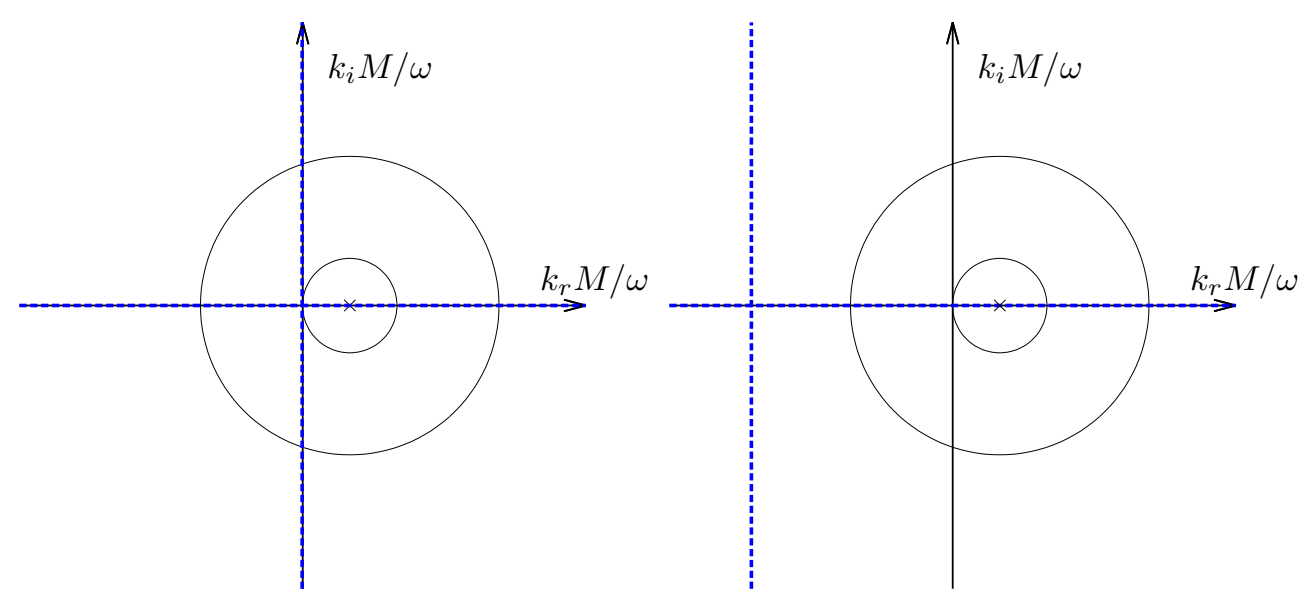

Figure 4: Illustration of the duct approximation of a cylindrical vortex sheet for $T=1$ and (a) $M=0.1$ and (b) $M=0.9$. The dashed blue lines represent locations in the complex $k$ plane of duct eigenvalues. The inner circle is $|k M / \omega-1|^{2}=1$, and the outer circle is $|k M / \omega-1|^{2}=10$. The cross marks $k M / \omega=1$.

Therefore, the dispersion relationship $\mathcal{D}_{j}=0$ is approximately satisfied by the duct mode if

$$
\left|1-\frac{k_{m, n}^{ \pm} M}{\omega}\right|^{2} \gg 1 .
$$

The parameter $1-k M / \omega$ can be expressed as $1-M / M_{\phi}$, where $M_{\phi}$ is a Mach number related to the complex-valued phase-speed. Condition (17) is satisfied if $\left|M_{\phi}\right| \ll M$, i.e. there is a scale separation between phase-speed and jet velocity. The effect of this condition can be seen by considering the vortex sheet displacement:

$$
\eta=\frac{T}{\omega^{2}\left(1-\frac{M}{M_{\phi}}\right)^{2}} \frac{d p_{i}}{d r}\left(\frac{1}{2}\right) .
$$

The vortex sheet displacement is maximal for $M_{\phi} \approx M$ and tends to zero if $\left|M_{\phi}\right| \ll M$. A second effect of condition (17) is that if $\left|M_{\phi}\right| \ll M$ we should also have $\left|M_{\phi}\right| \ll 1$ for subsonic and moderately supersonic jets; in this case, the phase speed of the duct mode is much smaller than the speed of sound, and the outer stream only supports evanescent waves with fast radial decay. Therefore, a soft duct mode satisfying equation (17) is an approximate solution to the vortex-sheet problem with nearly zero vortex-sheet displacement and rapidly decaying evanescent waves in the outer stream. The pressure is thus close to zero outside the jet, and these acoustic modes are trapped within the jet core.

Equation (17) is represented graphically in figure 4(a) and (b), which are drawn respectively for $M=0.1$ and $M=0.9$ isothermal $(T=1)$ jets. The possible values of duct wavenumbers are marked with dashed blue lines; evanescent modes are represented by the vertical line crossing the real axis at $-M^{2} /\left(1-M^{2}\right)$, and propagative modes should lie on the real axis. The inner circle represents positions satisfying $\left|k_{r} M / \omega-1\right|^{2}=$ 1 , and the outer corresponds to $\left|k_{r} M / \omega-1\right|^{2}=10$. If a mode for a soft duct lies well beyond the outer circle, condition (17) is satisfied, and this soft duct mode is an approximate solution for the cylindrical vortex sheet. There is a different behaviour for low and high subsonic Mach numbers. For low $M$, as in figure 4(a), evanescent soft duct modes with high decay rates provide reasonable approximations of the vortex sheet modes. However, as the frequency is increased, these modes approach the real axis, and no longer approximate a jet mode. On the other hand, for $M=0.9$ all evanescent modes satisfy (17) by at least an order of magnitude. The same cannot be affirmed for propagative modes: the $k^{-}$duct mode is always far from the outer circle, but the $k^{+}$mode approaches the circle as $\omega$ is increased, until a point where we expect condition (17) not to hold, such that the duct approximation can no longer be used. 


\section{Parallel-flow analysis}

In this section, we apply the parallel-flow stability theory to the three models to investigate the the waves in the potential core and the spectral tones in the near-nozzle region that are observed in the LES data ${ }^{2}$ for the Mach 0.9 isothermal jet.

\section{IV.A. Eigenvalues}

The spatial eigenvalues, $k=k(\omega)$, of the linearized Euler equations (at $x=0$ ), the vortex sheet, and the duct for $S t=0.3, m=0, M=0.9$, and $T=1$ are shown in figure 5 and serve as an introduction to the different families of modes that are observed at these flow conditions over a range of azimuthal wavenumbers and frequencies.

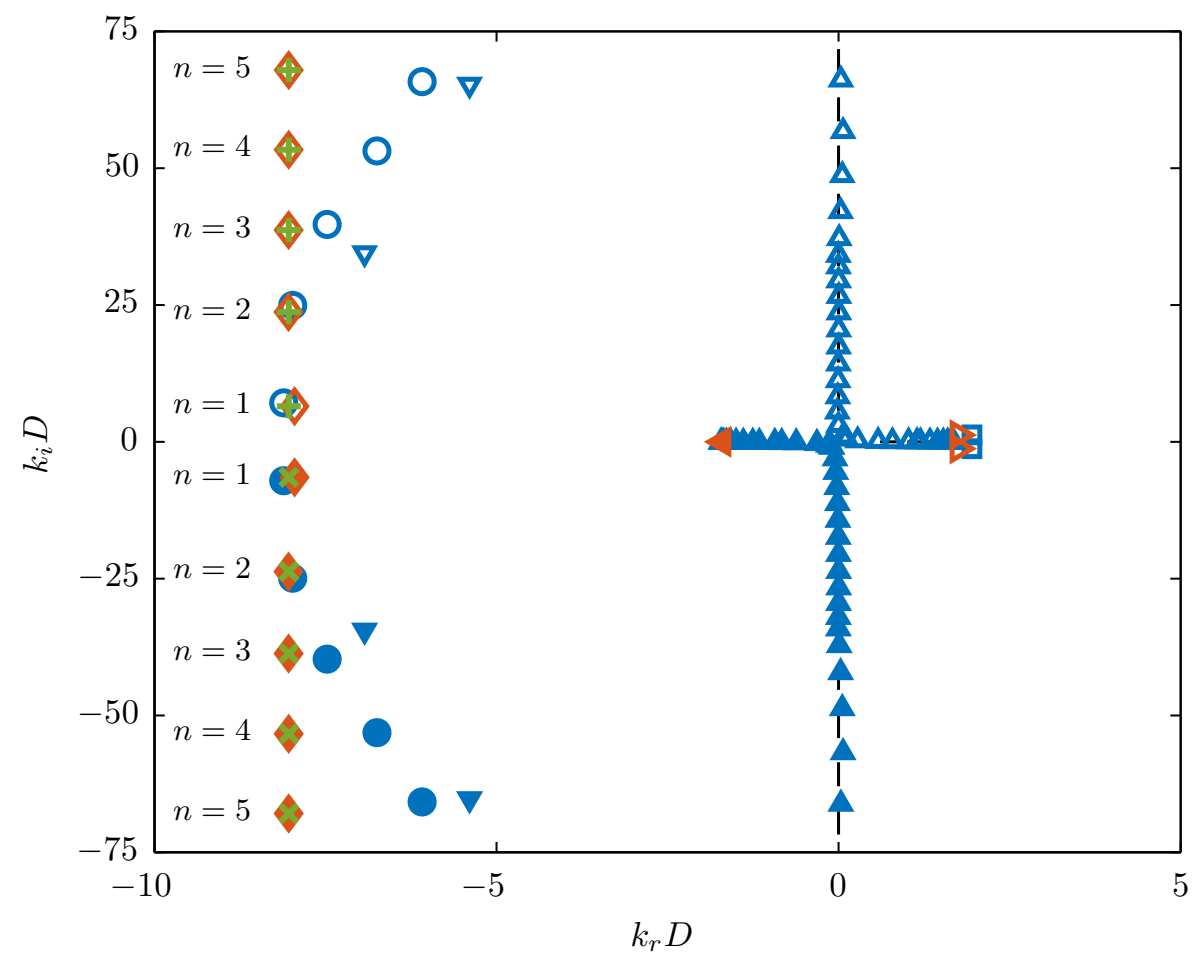

Figure 5: Eigenvalues for $S t=0.3$ and $m=0$. Linearized Euler modes: ( $\square$ ) Kelvin-Helmholtz; $(\Delta)$ down-

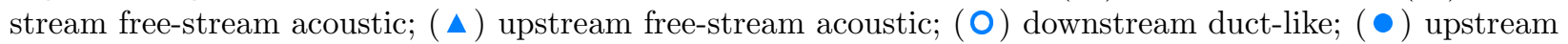
duct-like; additional downstream $(\nabla)$ and upstream $(\boldsymbol{\nabla})$ negative phase velocity modes. Vortex sheet modes: $(\Delta)$ Helvin-Helmholtz; ( $\triangleleft)$ upstream free-stream-like; $(\diamond)$ downstream duct-like; $(\diamond)$ upstream duct-like. Duct modes: $(+)$ downstream; $(x)$ upstream. The dashed lines show the continuous branch of eigenvalues for acoustic waves in an unbounded quiescent fluid.

First, the Euler and vortex sheet models support a single convectively unstable discrete mode (and its stable complex conjugate) that represents the well-known Kelvin-Helmholtz instability and corresponds to Michalke's Mode I. ${ }^{5}$ The Euler equations also supports additional stable convective modes (omitted from the figure to reduce clutter) that describe the transport of vorticity and entropy at the local mean velocity at each radial station.

Second, the Euler equations support two families of modes that represent the discrete approximation of continuous branches of upstream and downstream traveling free-stream acoustic modes. These modes approximately satisfy the dispersion relation for an acoustic wave in an unbounded quiescent fluid, which is shown as dashed lines in the figure. The horizontal and vertical parts of these branches represent propagative and evanescent acoustic waves, respectively. These modes have supersonic phase-speeds. The Euler equations and vortex sheet also support a discrete free-stream acoustic mode with subsonic phase-speed that lies just to the left of the locus of the downstream propagative free-stream acoustic branch and represents an acoustic 
wave the propagates directly upstream.

Third, all three models support discrete modes with large negative wavenumbers. The Euler modes marked by upside-down triangles have no counterpart in the vortex sheet and duct models, and we do not consider them further. The remaining modes with negative phase-speeds are similar in all three models. These modes appear in complex conjugate pairs. By applying the criteria from equations (5) and (6), the $k_{i}>0$ and $k_{i}<0$ modes can be shown to be downstream and upstream traveling, respectively. At this frequency, they are all evanescent, but we will see that each $(m, n)$ mode pair becomes propagative at certain frequencies. The mode pairs can be classified using two integers, $(m, n)$, where $m=0,1, \ldots$ is the azimuthal wavenumber and $n=1,2, \ldots$ is a radial mode number equal to the number of anti-nodes in the radial pressure distribution. The vortex sheet and duct eigenvalues are nearly identical because the duct modes satisfy condition (17). Therefore, the vortex sheet modes behave as duct-like modes. The equivalent Euler eigenvalues are well-approximated by the duct and duct-like votex sheet eigenvalues for low values of $n$, but diverge for higher values of $n$, but the higher modes are highly damped at this frequency. As mentioned already, each mode pair will become propagative at some frequency, at which point the discrepancy becomes small for every $n$. Therefore, the Euler modes can also be viewed as duct-like. The close correspondence of the vortex sheet and Euler modes to the duct modes indicates that they represent acoustic waves that experience the jet as a soft duct. These modes are part of Michalke's Mode II family, and we will show that they are related to the waves observed in the potential core. We will see that the duct-like and discrete free-stream modes interact with one-another in a number of important ways. To be concise, we will use the term core modes to refer to these different modes together as a group.

We next study the behavior of the core modes as a function of $\omega$. Figure 6 shows the path of the $(0,1)$ modes in the complex $k$ plane as $\omega_{r}$ is varied with different values of $\omega_{i}$ for each model. Focus first on the solid line, which corresponds to $\omega_{i}=0$. The downstream traveling eigenvalues start with positive imaginary part and move toward the real axis as $\omega_{r}$ is increased and the upstream traveling eigenvalues start with negative imaginary part and move toward the real axis as $\omega_{r}$ is increased. At some critical frequency, the two eigenvalues coalesce. Beyond this frequency, they move along the $k_{r}$ axis. The mode moving toward $k_{r}=0$ can be shown to be downstream propagating, while the mode moving away from $k_{r}=0$ is upstream propagating. As $\omega_{r}$ is further increased, the downstream vortex mode coalesces with a the third upstream propagating mode, and both modes move off the $k_{r}$ axis. The duct eigenvalues do not exhibit this behavior, but instead continue indefinitely along the real axis. The dashed lines correspond to $\omega_{i} \neq 0$, and show that the mode intersection points are saddle points. All of the saddle points occur between an upstream and downstream propagating mode and therefore meet the criteria from section II. Since they occur for $w_{i}=0$, they correspond to neutral solutions. That is, within a purely linear context, they would neither grow nor decay at their source, but persist indefinitely.
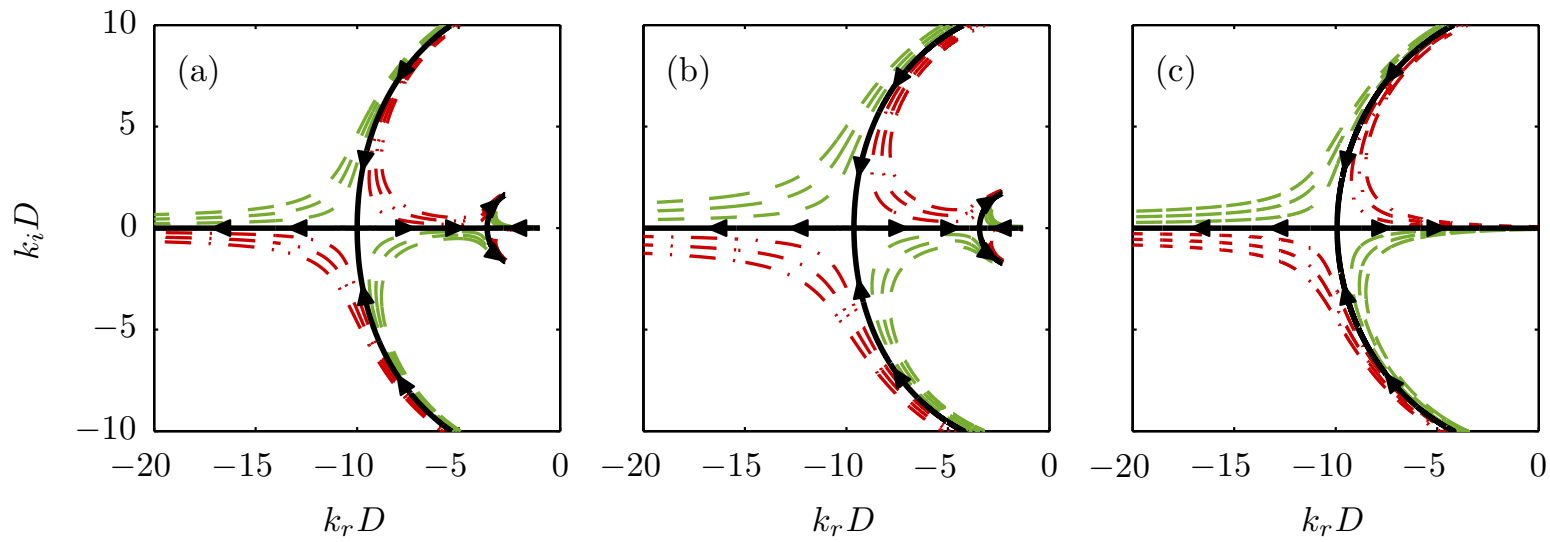

Figure 6: Saddle points for the $(0,1)$ mode group at $M=0.9$ and $T=1$ for: (a) the linearized Euler equations (at $x=0$ ); (b) the vortex sheet; (c) the soft duct. Lines: $(-) \omega_{i}=0 ;(---) \omega_{i}<0$; $(-\cdot-\cdot) \omega_{i}>0$. The arrows show the direction of increasing $\omega_{r}$. 


\section{IV.B. Dispersion relations}

The part of the $\omega_{i}=0$ curve that lies on the $k_{r}$ axis corresponds to propagating waves that convect away from their source without growing or decaying. For the first three radial modes at $m=0$, the dispersion relation for these propagating waves are plotted in figure 7 on top of the LES frequency-wavenumber diagram, which can be understood as an empirical dispersion relation for the waves in the potential core. The local minima and maxima in each Euler and vortex sheet dispersion relation correspond to the first and second saddle points, respectively. These dispersion relations closely match the bands of negative-wavenumber energy in the data. The duct dispersion relation provides a good approximation for wavenumbers to the left of the second saddle point, which is consistent with equation (17). The close match between the model and empirical dispersion relations shows that the core modes describe the waves observed within the potential core.

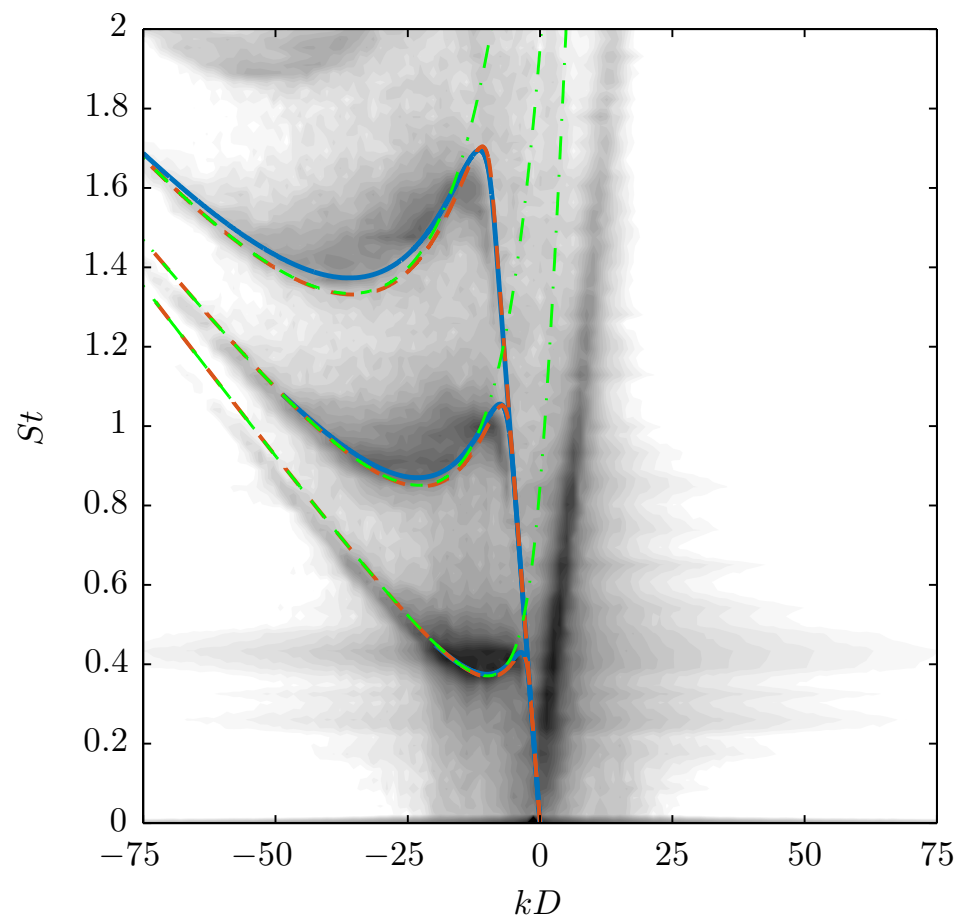

Figure 7: Comparison between modeled and empirical dispersion relations for the $m=0$ potential core waves: (- linearized Euler model; $\left(--_{-}\right)$vortex sheet model; $(-.-.-)$duct model. The contours show the empirical dispersion relation obtained via frequency-wavenumber decomposition of the pressure along the polar axis.

\section{IV.C. Tones}

Within the dispersion relation for each mode group, the waves in the LES data are particularly energetic at frequencies in between the two saddle points. At these frequencies, the jet supports both upstream propagating waves (negative slope in figure 7) and downstream propagating waves (positive slope). At other frequencies, the dispersion relations contain only upstream propagating waves. Therefore, for each radial mode group, there is a restricted frequency range that is bounded by the two saddle points for which there exist both upstream and downstream propagating core modes. We will see in the next section that this provides a necessary ingredient for resonance between the upstream and downstream propagating modes in the potential core at these frequencies. For now, we simply observe that these frequency ranges accurately predict the tonal peaks observed outside of the jet. The shaded regions in figure 8 indicate the range of frequencies between the two vortex-sheet saddle points for the first three radial mode groups at $m=0$ and 1 (the Euler model gives almost identical frequency bands). These frequency bands provide an accurate 
prediction of the tonal frequencies detected in the near-nozzle pressure field for both the LES and experiment data-sets.
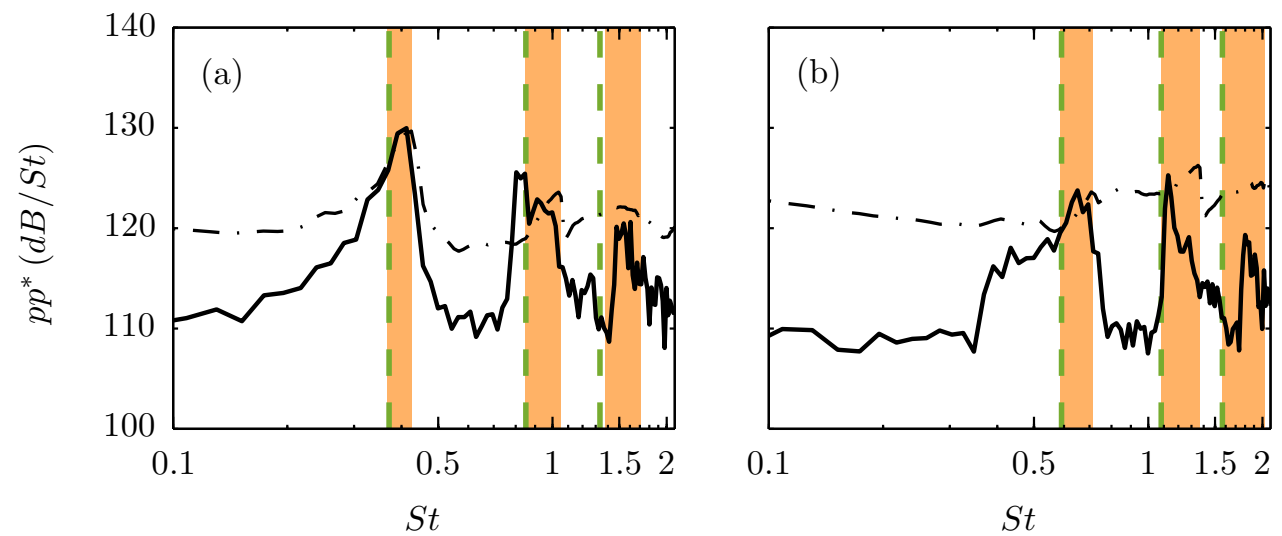

Figure 8: Power-spectral-density of the of pressure in the near-nozzle region $((x / D, r / D)=(0.02,0.8))$ : (a) $m=0$; (b) $m=1$. Data: (—) LES data $;^{1}(-\cdot-\cdot)$ experimental measurements. ${ }^{2}$ Model predictions: $(\square)$ frequencies between the two saddle points in the vortex sheet model; $(---)$ duct saddle point.

\section{IV.D. Eigenfunctions}

Next, we compare the radial structure of the core modes to the LES data at $x=0$. In anticipation of the key role of the aforementioned resonance, we make these comparisons for each $(m, n)$ mode group at a frequencies between the two saddle points. Each column in figure 9 makes this comparison for one mode group. The rows, from top to bottom, show model predictions for the upstream and downstream propagating duct (or duct-like modes) and the upstream propagating free-stream mode. For each case, the squared magnitude of the pressure component of the eigenfunction from each model is compared to the power-spectral density of the LES pressure fluctuation at the jet inlet (the data is the same in each row). The data and eigenfunctions have been normalized to have a maximum value of one.

Within the potential core, the upstream and downstream propagating duct (or duct-like) modes for the three models are indistinguishable from one another, and also match the LES data over a range of two orders-of-magnitude. Outside of the potential core, the data falls off at a rate that is much slower than the duct modes, but that is accurately captured by the free-stream mode. Therefore, a linear combination of the duct modes and the free-stream mode can account for the radial shape of the power-spectral-density of the LES data at $x=0$ both inside and outside of the core. This match further confirms that the core modes describe the waves in the data and indicates that they dominate the pressure field near the jet inlet. 

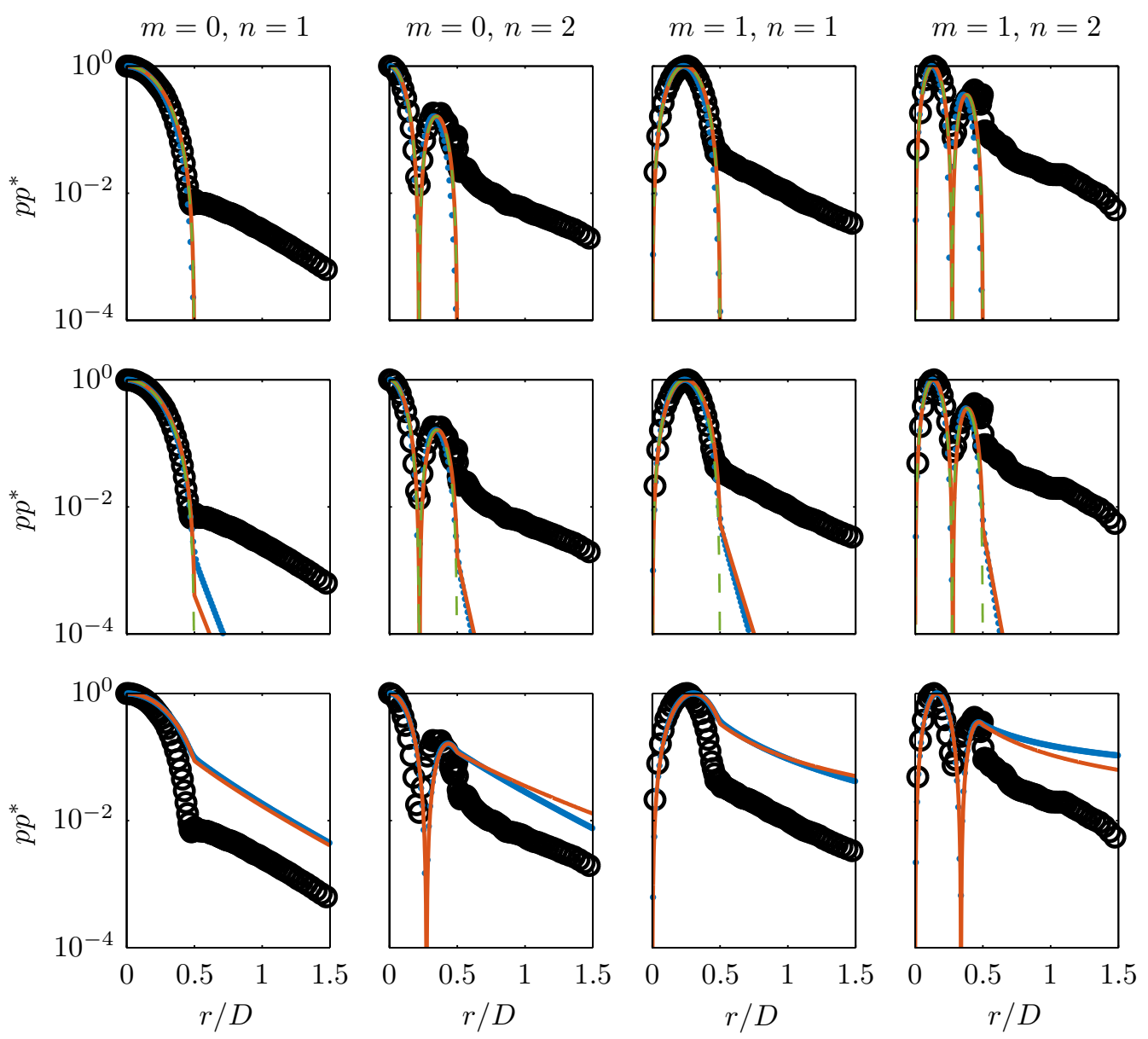

Figure 9: Radial form of the power-spectral-density of the pressure at $m=0$ : ( O) LES data; $(\bullet)$ linearized Euler model; (- $(-$ vortex sheet model; $(---)$ duct model. Columns (left to right): $m=0, S t=0.4$; $m=0, S t=0.96 ; m=1, S t=0.66 ; m=0, S t=1.24$. Rows (top to bottom): the upstream and downstream propagating duct (or duct-like) modes and the upstream propagating free-stream mode. The LES data is the same in each row. 


\section{IV.E. Mach number effects}

Next, the vortex sheet and duct models are used to explore the Mach number dependence of the trapped modes at a fixed temperature ratio. Specifically, the $(k, \omega)$ locations of the vortex sheet and duct saddle points are calculated as a function of the Mach number at $T=1$. The results for $m=0, n=0$ are shown in Figure 10.
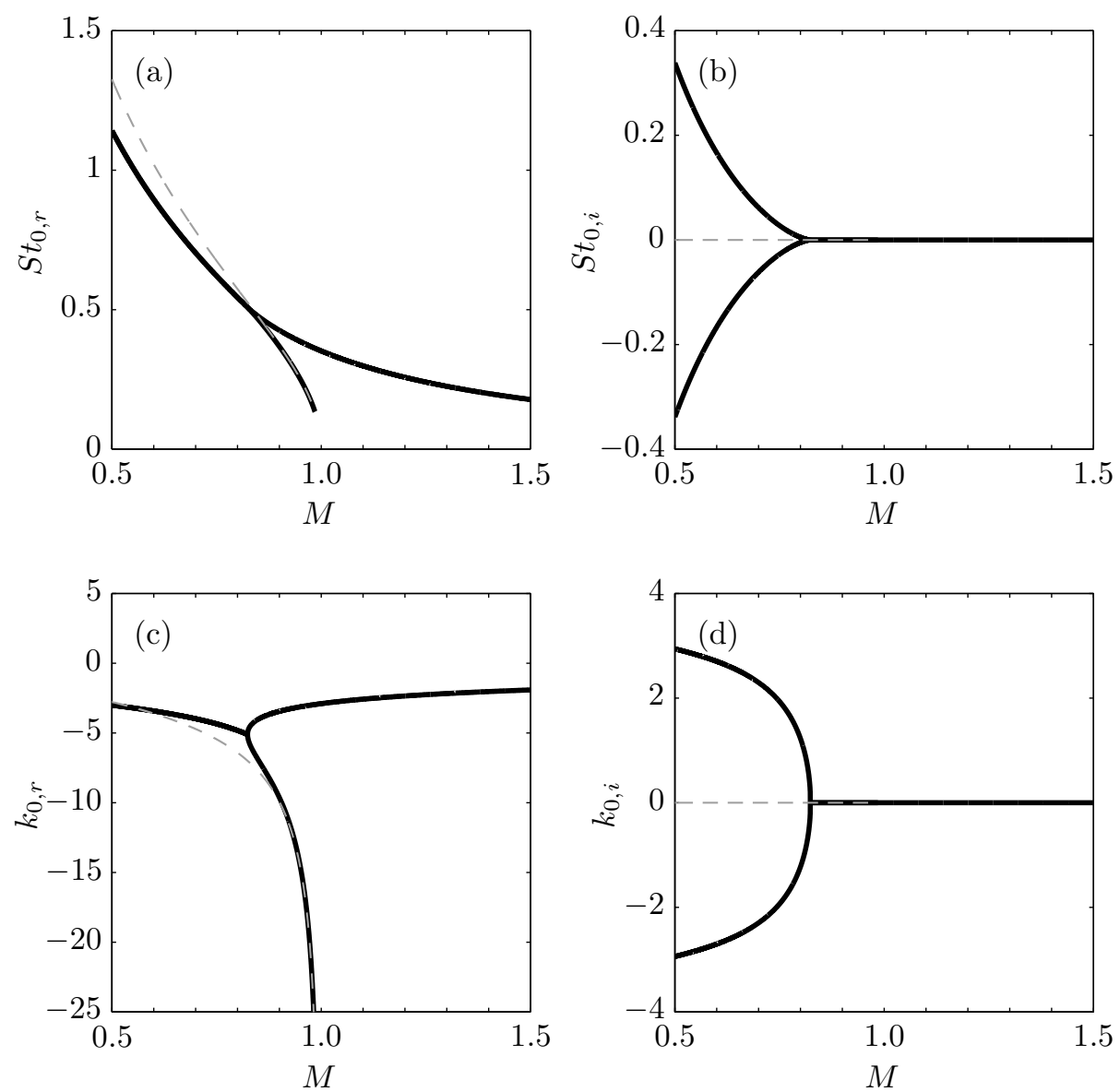

Figure 10: Saddle point locations for the $(0,1)$ mode group as a function of Mach number for the vortex sheet (- $)$ and the duct analogy $(---)$.

At $M=0.9$, the vortex sheet modes form two saddle points as shown in the previous subsections. Moving toward smaller Mach numbers, a pitchfork bifurcation is encountered at $M=0.82$ at which the two saddles merge and move off the real $k$ and $\omega$ axes. The saddle with $\omega_{i}>0$ occurs between two upstream modes and is therefore irrelevant from a stability standpoint. The saddle with $\omega_{i}<0$ occurs between an upstream mode and downstream mode, and is therefore valid. As a result, the modes are absolutely stable for $M<0.82$. Moving from $M=0.9$ toward higher Mach numbers, we encounter a different kind of bifurcation at $M=1$. The second saddle continues on the real axes unaffected by the switch from subsonic to supersonic flow, but the first saddle asymptotically approaches zero frequency and a negative infinite wavenumber. Therefore, for $M>1$, there is one saddle on the real axes.

The number of saddles on the real axes - zero for $M<0.82$, two for $0.82<M<1$, and one for $M>1$ - has a profound impact on the types of propagating waves supported by the flow. Specifically, these saddle points represent points of zero group velocity that act as transition points between upstream and downstream propagation, so the number of saddle points determines the maximum possible number of upstream and downstream propagating waves. Figure 11 shows the dispersion relation of the $m=0, n=0$ propagating waves for $M=0.6$ and $M=1.5$, which should be compared to the $M=0.9$ results from figure 7. As we've seen, for $M=0.9$ there is a distinct frequencies range, bounded by the two saddle points, 

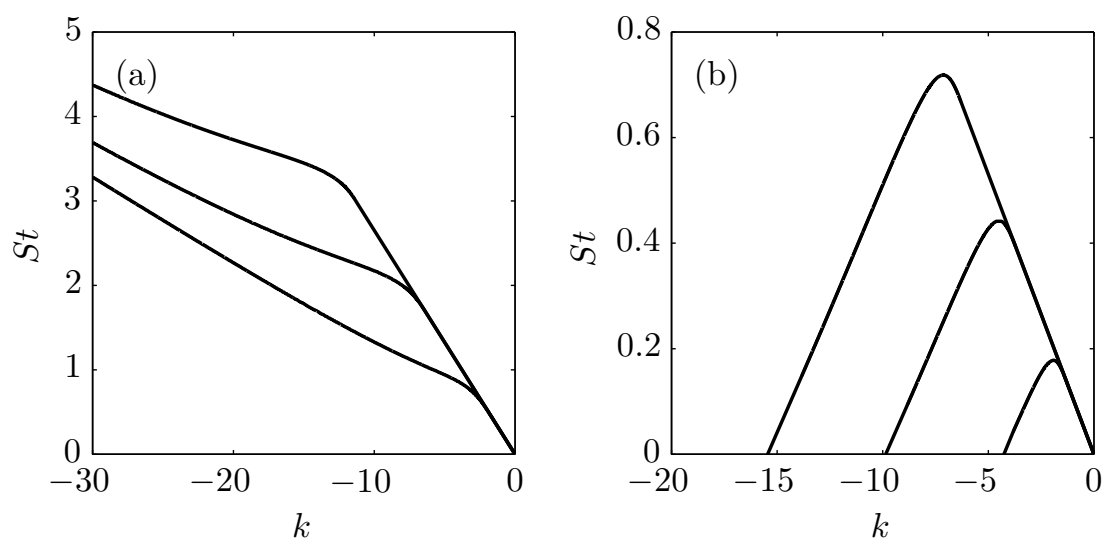

Figure 11: Neural curves for the first three $m=0$ radial modes of the vortex sheet at $T=1$ and (a) $M=0.6$ and (b) $M=1.5$. The analogous curves for $M=0.9$ appear in figure 7 .

for which both upstream and downstream propagating trapped waves exist, while only upstream waves are supported at other frequencies. For $M=0.6$, there exist only upstream propagating trapped waves at all frequencies since there are no saddle points on the real axes. For $M=1.5$, there exist both upstream and downstream trapped waves at all frequencies due to the presence of a single saddle point on the real axis. Our results at $M=0.6$ and $M=1.5$ are consistent with the findings of Tam \& Hu. ${ }^{16}$ They referred to the trapped modes described in this paper as "subsonic instability modes," and noted that only upstream waves exist for $M=0.6$ while both upstream and downstream waves exist for $M=1.5$, as well as other higher Mach numbers. However, they did not investigate the transition between these two cases and consequently missed the two-saddle-point region.

As already mentioned, the existence of both upstream and downstream propagating trapped waves at the same frequency leads to the possibility of resonance. For $M<0.82$, no such frequencies exist. For $0.82<M<1$, there are limited frequency bands, bound by the two saddle points, that have this property. This leads to the generation of tones at these frequencies, as shown in figure 8 . For $M>1$, there are both upstream and downstream propagating waves at all frequencies, resulting in broadband energy distribution rather than discrete tones. To summarize, only jets within a limited Mach number range, $0.82<M<1$ for $T=1$, have two saddle points on the real axes, which is a key ingredient for generating tones.

\section{IV.F. Temperature ratio effects}

Finally, the temperature ratio dependence of the trapped modes is incorporated into the analysis with the goal of determining certain Mach number, temperature ratio combinations that support two saddle points on the real axis. Specifically, the two Mach number bifurcation points described in the previous subsection for $T=1$ are computed for a range of temperature ratios.

The results are summarized for $m=0$ and $m=1$ in figure 12. Recall that $M=U_{j} / c_{\infty}$ is the acoustic Mach number. The back curve represents the supersonic bifurcation point, which is exactly described by the relation $M=\sqrt{T}$, which corresponds to a jet Mach number $M_{j}=U_{j} / c_{j}=1$. This bifurcation point is exactly predicted by the duct modes and the boundary is independent of $n$. On the other hand, the location of the subsonic bifurcation is a weak function of $n$. The dark blue curve, light blue curve, and green curve show $n=0, n=1$, and $n=2$, respectively. The spacing between the curves decreases with increasing $n$, suggesting the existence of an asymptotic curve that bounds the region for all $n$. The grey region demarcates the Mach number, temperature ratio combinations between the two bifurcation points (up to $n=3$ ). For these values, two saddle points exist and spectral tones are therefore expected to be observed.

These predictions can be compared to experimental and numerical observations. The red and black symbols represent set-points where tones have and have not been observed, respectively. The circles correspond to results from Suzuki \& Colonius ${ }^{3}$ while the squares correspond to two LES databases, one of which is the Mach 0.9 isothermal jet used throughout the paper. ${ }^{2}$ The agreement is good, with only one set-point 

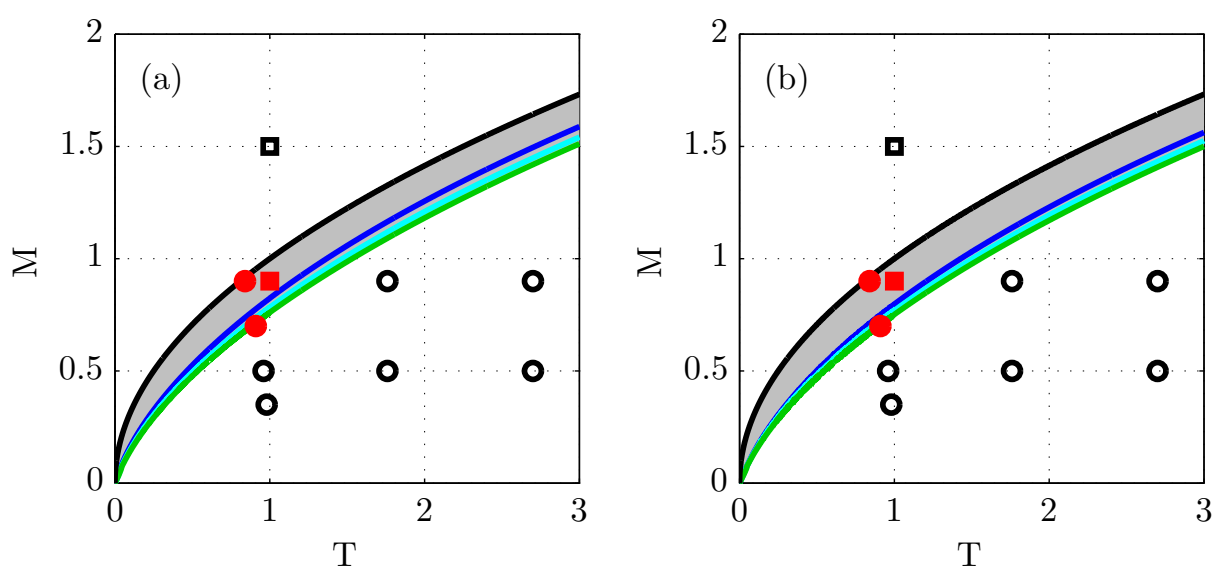

Figure 12: Saddle point Mach number bifurcations as a function of temperature ratio for (a) $m=0$ and (b) $m=1$. Black curve: supersonic bifurcation. Other curves: subsonic bifurcation. Shaded region: operating conditions where the model predicts the existence of tones. Closed symbols: jets in which tones have been observed. Open symbols: jets in which tones have not been observed. Squares: Computational jets. Circles: experimental jets.

disagreeing with the model. For this point, tones are observed dispite being slightly below the subsonic bifurcation point. This is consistent with recent results of Jaunet et. al, ${ }^{17}$ who found that the tones gradually disappear as the Mach number is decreased below the subsonic bifurcation point rather than suddenly vanishing. This can be explained by the fact that the trapped waves are lightly damped at Mach numbers just below the bifurcation point and gradually become more damped with decreasing Mach number (see figure 10).

\section{Weakly-nonparallel analysis and resonance}

So far, we've shown that one of the ingredients for resonance of the trapped waves, the existence of both upstream and downstream propagating core modes, exists within limited frequency bands for jets with high subsonic Mach numbers. For resonance to occur, a pair of end conditions capable of linking together the upstream and downstream propagating waves are also required. One end condition is provided by the nozzle, which can be viewed as an impedance boundary condition leading to a matrix of reflection coefficients that describes the redistribution of energy from each upstream traveling wave that hits the boundary into every possible downstream traveling wave. If the reflection coefficient between the upstream and downstream propagating trapped waves is nonzero, then part of the incident upstream propagating wave is converted into a downstream propagating trapped wave. In this section, we will show that the second end condition for resonance is provided by the slow streamwise contraction of the potential core.

To this end, we use a weakly-nonparallel extension of the previous parallel-flow spatiotemporal theory based on a WKB ansatz of the form

$$
q(x, t) \sim A(X) \exp \left[\frac{\mathrm{i}}{\epsilon} \int_{0}^{X} k(\omega ; X) d X-\mathrm{i} \omega t\right],
$$

where $\epsilon$ is a small parameter that typifies the slow streamwise variation of the flow, $X=\epsilon x$ is the slow streamwise variable, and $A(X)$ is an unknown amplitude function. After some analysis, the complex wavenumber $k(\omega, X)$ can be shown to be the solution of a local dispersion relation

$$
\mathcal{D}(k, \omega ; X)=0
$$

obtained by freezing the coefficients of the slowly-varying equations at each $x$ (and again neglecting streamwise derivatives of the mean flow as well as the mean transverse velocity). ${ }^{18}$ We will apply this weakly- 

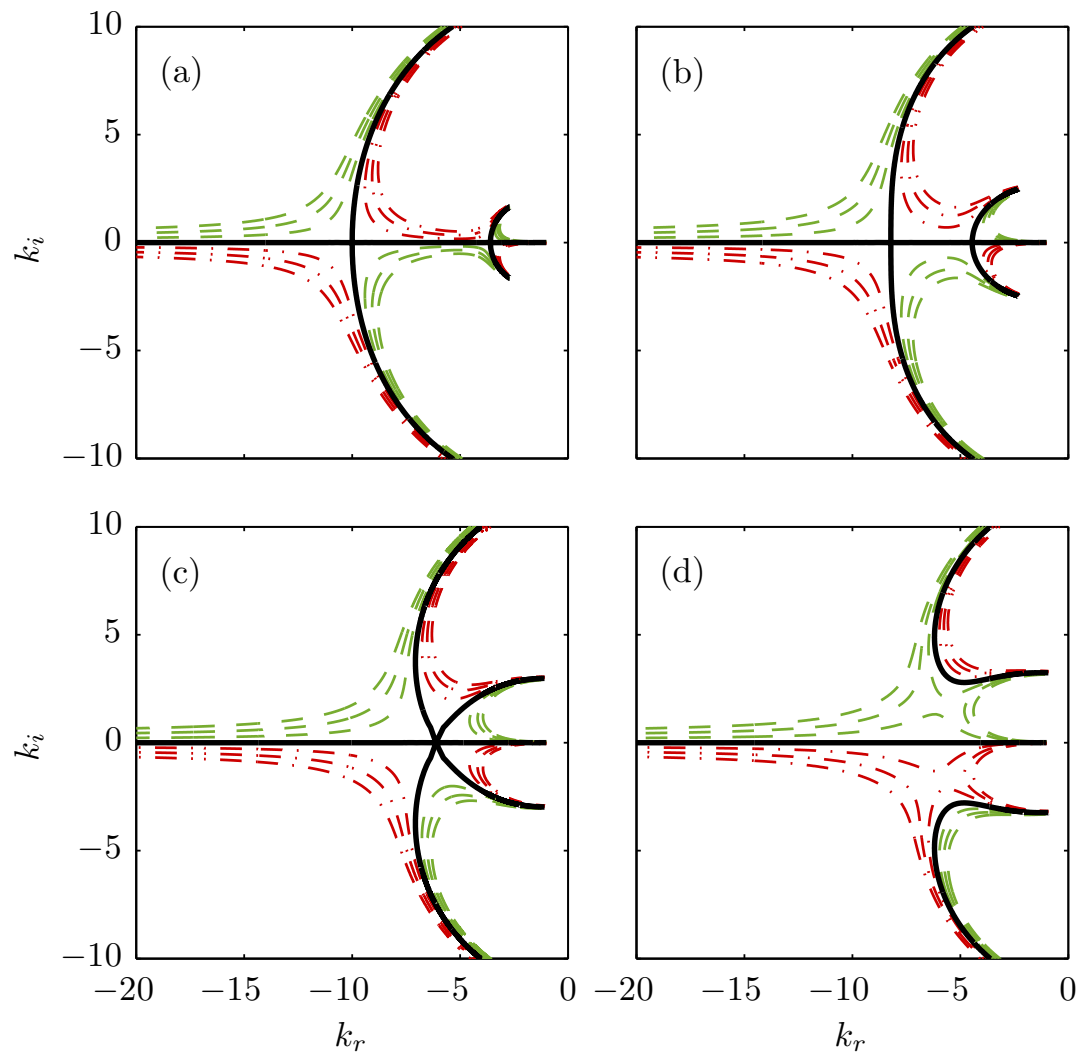

Figure 13: Saddle points of the LES mean-flow at $m=0$ and (a) $x=0$, (b) $x=3$, (c) $x=3.62$ and (d) $x=4$. The line types correspond to: (- $) \omega_{i}=0 ;(---) \omega_{i}<0 ;(-\cdot-\cdot) \omega_{i}>0$. The two saddle points that occur on the real axis for low $x$ merge at $x=3.62$ and subsequently move off of the real axis.

nonparallel theory to the Euler equations linearized about the spatially evolving mean jet, and in particular to the the core modes identified in the previous sections.

The amplitude function, which is determined by matching terms at $\mathcal{O}(\epsilon)$, is singular at so-called turning points of the WKB approximation. ${ }^{18}$ These turning points represent special locations in the complex $(k, \omega, X)$ space at which upstream and downstream solutions of the local dispersion relation can exchange energy and be stitched together to form an approximate global solution at a given frequency. Specifically, at a turning point, an incident downstream propagating wave is split into a reflected wave that is upstream propagating and an evanescent transmitted wave.

These turning points occur at locations of zero group velocity, which again correspond to saddle points in the complex $k$-plane. Therefore, the saddle points not only govern the stability properties of the flow, ${ }^{19}$ but also provide the second end condition for resonance. Our task, then, is to track the two saddle points identified in the previous section as a function of $x$. The saddles for the $(0,1)$ mode group are shown in figure 13 for four streamwise locations. As $x$ increases, the two saddle points that were identified at $x=0$ slowly deform and move towards each other along the $k_{r}$-axis. The absolute frequency remains real-valued for both saddle points. They continue this trajectory until they coalesce at $x / D=3.62$, forming a second-order saddle point. Beyond this critical $x$ location, both saddle points move off the $k_{r}$-axis, and the associated absolute frequency becomes complex-valued. The upper saddle point occurs for $w_{i}<0$ and is therefore absolutely stable. The lower saddle point occurs for $w_{i}>0$, but both participating modes can be shown to be upstream traveling, making the saddle point irrelevant, as discussed in Section II. Therefore, the $(0,1)$ core modes are neutrally stable for $x \leq 3.62$ and absolutely stable for $x>3.62$. As was the case in Section IV.E, when the saddle points moved off of the real axis, the jet no longer supports downstream propagating trapped waves. Similar behavior is observed for other $(m, n)$ mode groups, but the absolute frequencies and wavenumbers associated with the saddle points are different, as are the critical $x$ value at 

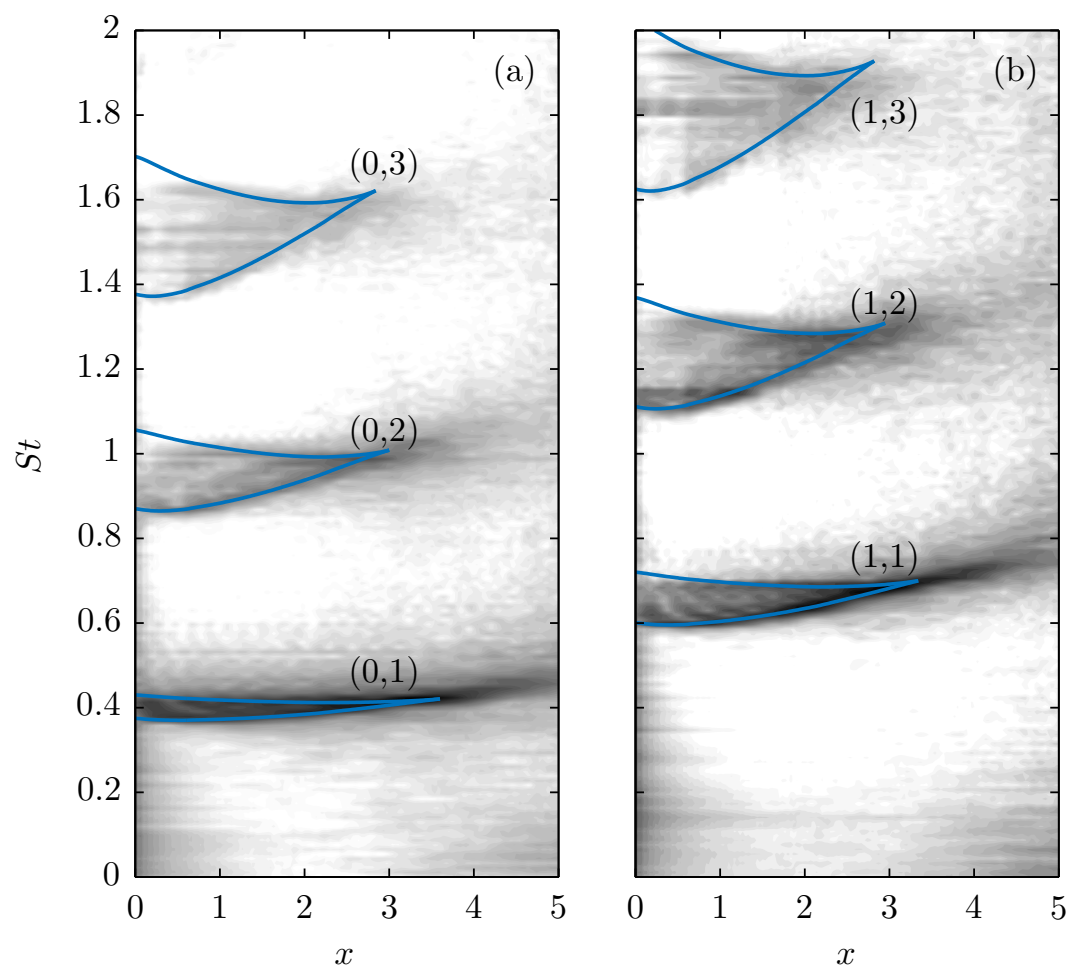

Figure 14: Power-spectral-density of the phase-speed-filtered pressure: (a) $m=0, r / D=0$; (b) $m=1$, $r / D=0.1$. The contours levels are distributed on a logarithmic scale spanning four orders-of-magnitude. The lines show the locations of the WKB turning points.

which they coalesce and become absolutely stable.

The blue lines in figure 14 give the frequencies for the two saddle points as a function of $x$ up until the critical merger point for several $(m, n)$ mode groups (the grayscale contours will be discussed later). There are two lines for each $(m, n)$ that correspond to the position of the two saddle points. The two lines necessarily intersect at the streamwise location at which the two saddle points coalesce. Recall that, at each $x$, the two saddle points bound the frequencies and wavenumbers for which both upstream and downstream propagating trapped waves exist. Therefore, for each $(m, n)$, both upstream and downstream propagating waves exist only for frequencies and wavenumbers on the interior of the region bounded by the two $S t^{0}(x)$ curves and the line $x=0$.

An end condition for resonance exists at $x=x_{t}$ for a wave in the $(m, n)$ mode group if its Strouhal number satisfies the condition $S t=S t^{0}\left(x_{t}\right)$ for one of the two $(m, n)$ saddle points. This condition can be visualized by drawing a horizontal line at the desired frequency in figure 14. A turning point exists at that frequency for mode group $(m, n)$ if the horizontal line intersects one of the $S t^{0}$ curves for that mode group, and the streamwise location of the turning point is given by the $x$ value of the intersection point. This happens only for frequencies that support both upstream and downstream trapped waves. It's interesting to note that the turning points occur well before the end of the potential core at $x / D \approx 6$. By analogy with a spatially varying, cylindrical hard-walled duct, for which similar turning points arise when the cross-section decreases with $x,{ }^{20}$ the jet turning points can be attributed to the gradual streamwise contraction of the potential core.

The repeated reflection of the trapped waves between the end conditions provided by the nozzle and the turning points leads to a true resonance only if the upstream and downstream traveling waves mutually reinforce one another by interfering constructively rather than destructively. This is the case only if certain conditions on the wavenumbers and reflection coefficients of the waves are met. Rather than using the weakly-nonparallel theory, we address this question in a parallel investigation using global modes of the jet. ${ }^{21}$ This analysis confirms that the conditions for resonance are met for certain discrete frequencies. 
Finally, we return to the LES data to assess whether it shows evidence of resonance between the nozzle and the turning points of the WKB solution. Whereas our theoretical model is meant to describe the core waves only, the data is made up of convective disturbances in addition to the acoustic core waves (see, for example, figure 2). To foster a fair comparison, we use the frequency-wavenumber decomposition discussed previously as a filter to isolate the core waves. The key insight is that the core waves have negative phase-speeds while the dominant convective instabilities have positive phase-speeds. Therefore, zeroing the positive wavenumber components and taking an inverse streamwise Fourier transform filters out all waves with positive phase-speeds, effectively distilling the negative phase-speed core waves. Contours of the powerspectral-density of the $m=0$ and 1 components of the the phase-speed-filtered pressure at $r / D=0$ and 0.1 , respectively, are plotted in figure 14. The contours levels are distributed on a logarithmic scale spanning four orders-of-magnitude. This visualization of the data makes it clear that the core waves are energetic primarily on the interior of the region bound by the $S t^{0}$ and $x=0$ curves, i.e., regions of $x-S t$ space that support a resonance of propagating waves.

\section{Summary and conclusions}

In summary, using modal analysis of the linearized Euler equations, a cylindrical vortex sheet, and a cylindrical soft duct, we have shown that the waves observed within the potential core of subsonic jets are resonating acoustic waves that are trapped within the potential core. The propagating waves and end conditions necessary for this resonance exist only within limited frequency bands that are bound by two saddle points formed by the linear modes that describe the waves, leading to tones that can be detected outside of the jet in the near-nozzle region. Furthermore, the vortex sheet model predicts that these resonant frequency bands exist only for certain combination of Mach number and temperature ratio, which explains why tones are observed in some jets but not others.

Our models capture with striking accuracy numerous aspects the trapped waves that are extracted from LES data of a Mach 0.9 isothermal jet. The model dispersion relations closely follow the empirical dispersion relations obtained through frequency-wavenumber decomposition of the data. The frequency bands between the two saddle points predict the tonal frequencies. The radial structure of the pressure field near the nozzle is described by the eigenfunctions of the core modes. The identified resonance mechanism provides predictions for the frequency and spatial energy distribution for the trapped waves that closely match the data. These results confirm that the models capture the essential physics of the potential core waves.

Whether or not these trapped waves are important to the overall jet dynamics or acoustics remains to be established. Our parallel global analysis ${ }^{21}$ indicates that the resonating trapped waves are also weakly coupled to Kelvin-Helmholtz instability waves but do not play a significant role in the peak low-angle acoustic radiation. The role of the tapped waves is also being investigated in an ongoing experimental campaign. ${ }^{17}$ The findings suggest that the trapped waves can be observed in the far-field acoustic sprectrum at angles nearly tangent to the jet. The same experimental investigation also suggests that the trapped waves are responsible for strong tones that are observed in the acoustic field when a solid edge is introduced near the jet. Previous attempts ${ }^{22,23}$ to explain these edge tones did not yielded an accurate model, and the results of our analysis could provide the building blocks needed to do so. ${ }^{17}$

\section{Acknowledgments}

AT and TC gratefully acknowledge support from the Office of Naval Research under contract N0014-111-0753. AVGC and PJ acknowledge support from the Science Without Borders program (project number A073/2013). OTS was supported by DFG grant no. 3114/1-1. The LES study was supported by NAVAIR SBIR project, under the supervision of Dr. John T. Spyropoulos. The main LES calculations were carried out on CRAY XE6 machines at DoD HPC facilities in ERDC DSRC. 


\section{References}

${ }^{1}$ Brès, G. A., Jordan, P., Colonius, T., Rallic, M. L., Jaunet, V., and Lele, S., "Large eddy simulation of a turbulent Mach 0.9 jet," Tech. rep., Proceedings of the Center for Turbulence Research summer program, 2014.

${ }^{2}$ Brès, G. A., Jaunet, J., Le Rallic, M., Jordan, P., Colonius, T., and Lele, S., "Large eddy simulation for jet noise: the importance of getting the boundary layer right," 21sst AIAA/CEAS Aeroacoustics Conference, 2015.

${ }^{3}$ Suzuki, T. and Colonius, T., "Instability waves in a subsonic round jet detected using a near-field phased microphone array," J Fluid Mech., Vol. 565, 2006, pp. 197-226.

${ }^{4}$ Sinha, A., Development of reduced-order modes and strategies for feedback control of high-speed axisymmetric jets, Ph.D. thesis, The Ohio State University, 2011.

${ }^{5}$ Michalke, A., "Instabilitat eines kompressiblen runden Freistrahls unter Beriicksichtigung," 1971.

${ }^{6}$ Michalke, A., "A Note on the Saptial Jet-instability of the Compressible Cylindrical Wortex Sheet," Zentralstelle für Luftfahrtdokumentation und-information, 1970.

${ }^{7}$ Crighton, D. G., "Basic principles of aerodynamic noise generation," Progress in Aerospace Sciences, Vol. 16, No. 1, 1975, pp. 31-96.

${ }^{8}$ Briggs, R. J., Electron-Stream Interactions with Plasmas, MIT Press, Cambridge, Massachusetts, 1964.

${ }^{9}$ Bers, A., "Space-time evolution of plasma instabilities-absolute and convective," Basic plasma physics. 1, 1983.

${ }^{10}$ Towne, A. and Colonius, T., "Continued development of the one-way Euler equations: application to jets," Proceedings of the 20th AIAA/CEAS Aeroacoustics Conference, AIAA Paper 2014-2903, Atlanta, Georgia, June 2014.

${ }^{11}$ Strand, B., "Summation by parts for finite difference approximations for d/dx," J. Comput. Phys., Vol. 110, 1994, pp. 47-67.

${ }^{12}$ Colonius, T. and Ran, H., "A super-grid-scale model for simulating compressible flow on unbounded domains," J. Comput. Phys., Vol. 182, No. 1, 2002, pp. 191-212.

${ }^{13}$ Thompson, K. W., "Time Dependent Boundary Conditions for Hyperbolic Systems," J. Comput. Phys., Vol. 68, 1987, pp. $1-24$.

${ }^{14}$ Mohseni, K. and Colonius, T., "Numerical treatment of polar coordinate singularities," J. Comput. Phys., Vol. 157, No. 2, 2000, pp. 787-795.

${ }^{15}$ Lessen, M., Fox, J. A., and Zien, H. M., "The instability of inviscid jets and wakes in compressible fluid," J. Fluid Mech., Vol. 21, 1965, pp. 129-143.

${ }^{16}$ Tam, C. K. W. and Hu, F. Q., "On the three families of instability waves of high-speed jets," J. Fluid Mech., Vol. 201, 1989, pp. 447-483.

${ }^{17}$ Jaunet, V., Jordan, P., Cavalieri, A. V. G., Towne, A., Colonius, T., Schmidt, O., and Brès, G. A., "Tonal dynamics and sound in subsonic turbulent jets," Proceedings of the 22nd AIAA/CEAS Aeroacoustics Conference, Lyon, France, 2016.

${ }^{18}$ Bender, C. M. and Orszag, S. A., Advanced mathematical methods for scientists and engineers I, Springer Science \& Business Media, 1999.

${ }^{19}$ Huerre, P. and Monkewitz, P. A., "Local and global instabilities in spatially developing flows," Annu. Rev. of Fluid Mech., Vol. 22, 1990, pp. 473-537.

${ }^{20}$ Rienstra, S. W., "Sound propagation in slowly varying lined flow ducts of arbitrary cross-section," J. Fluid Mech., Vol. 495, 2003, pp. 157-173.

${ }^{21}$ Schmidt, O. T., Towne, A., Colonius, T., Jordan, P., Jaunet, V., Cavalieri, A. V. G., , and Brès, G. A., "Super- and multi-directive acoustic radiation by linear global modes of a turbulent jet," Proceedings of the 22nd AIAA/CEAS Aeroacoustics Conference, Lyon, France, 2016.

${ }^{22}$ Lawrence, J. and Self, R. H., "Installed jet-flap impingement tonal noise," Proceedings of the 21st AIAA/CEAS Aeroacoustics Conference, AIAA Paper 2015-3118, Dallas, Texas, 2015.

${ }^{23}$ Zaman, K. B. M. Q., Fagan, A. F., Bridges, J. E., and Brown, C. A., "Investigating the feedback path in a jet-surface resonant interaction," Proceedings of the 21st AIAA/CEAS Aeroacoustics Conference, AIAA Paper 2015-2999, Dallas, Texas, 2015. 